\title{
Surgery of primary non-small cell lung cancer with oligometastasis: analysis of 172 cases
}

\author{
Zhe Wang ${ }^{1}$, Shu-Geng Gao ${ }^{2}$, Qi Xue ${ }^{2}$, Xiao-Tong Guo ${ }^{2}$, Li-Xu Wang ${ }^{1}$, Xin Yu ${ }^{1}$, Yi-Kun Yang ${ }^{1}$, Ju-Wei Mu ${ }^{1,2}$ \\ ${ }^{1}$ Department of Thoracic Surgery, National Cancer Center/National Clinical Research Center for Cancer/Cancer Hospital \& Shenzhen Hospital, \\ Chinese Academy of Medical Sciences and Peking Union Medical College, Shenzhen 518116, China; ${ }^{2}$ Department of Thoracic Surgical Oncology, \\ National Cancer Center/National Clinical Research Center for Cancer/Cancer Hospital, Chinese Academy of Medical Sciences and Peking Union \\ Medical College, Beijing 100021, China \\ Contributions: (I) Conception and design: JW Mu, Z Wang; (II) Administrative support: JW Mu; (III) Provision of study materials or patients: \\ SG Gao, Q Xue; (IV) Collection and assembly of data: X Yu, YK Yang; (V) Data analysis and interpretation: Z Wang, XT Guo, LX Wang; (VI) \\ Manuscript writing: All authors; (VII) Final approval of manuscript: All authors. \\ Correspondence to: Ju-Wei Mu. Department of Thoracic Surgery, National Cancer Center/National Clinical Research Center for Cancer/ \\ Cancer Hospital \& Shenzhen Hospital, Chinese Academy of Medical Sciences and Peking Union Medical College, Shenzhen 518116, China. \\ Email: mujuwei@cicams.ac.cn.
}

Background: Previous studies have demonstrated that survival of patients with non-small cell lung cancer (NSCLC) with oligometastasis may benefit from local treatment. The purpose of this study was to compare the efficacy of local surgical treatment with systematic chemoradiotherapy in NSCLC with oligometastasis.

Methods: Data from a total of 172 patients with NSCLC with oligometastasis were collected at our Cancer Hospital from January 2006 to December 2016. The patients were divided into two groups: group A (82 cases) underwent primary surgical treatment and adjuvant chemotherapy was performed after operation, while group B (90 cases) received systematic chemotherapy and local radiotherapy. The median survival time (MST) and the 5-year survival rate of the two groups were compared and analyzed. The effects of various pathological types, surgical methods of the primary tumors and the site of oligometastasis were also analyzed. Results: The MSTs in groups A and group B were 48 months and 18 months, respectively, and the 5 -year survival rates were $21.1 \%$ and $7.6 \%$, respectively $(\mathrm{P}<0.05)$. In group $\mathrm{A}$, the survival rates were higher in patients with adrenal metastasis than patients with metastasis in the brain, bone, the liver or in other oligometastatic patients $(\mathrm{P}<0.05)$. There was no significant difference in the survival rate among the various pathological types or surgical methods of primary tumors $(\mathrm{P}>0.05)$.

Conclusions: Local surgical treatment of primary lesions in NSCLC significantly prolonged overall survival and 5-year survival rates of patients with NSCLC with oligometastasis.

Keywords: Non-small cell lung cancer (NSCLC); local surgical treatment; oligometastasis

Submitted Apr 23, 2018. Accepted for publication Nov 16, 2018.

doi: $10.21037 /$ jtd.2018.11.125

View this article at: http://dx.doi.org/10.21037/jtd.2018.11.125

\section{Introduction}

Primary non-small cell lung cancer (NSCLC) with oligometastasis has become a serious public health problem affecting the public's physical and mental health (1-4). In 2017, Siegel et al., Master of Public Health (MPH), and the American Cancer Society of Atlanta reported an estimated 222,500 cases of lung cancer and bronchogenic carcinoma, and the death toll was estimated at 155,870 (5). The mortality rate of lung cancer was the highest among all cancers, accounting for $16 \%$ (6) of all cancer patients. NSCLC accounts for $85 \%$ to $90 \%$ of lung cancer, and most of these patients have advanced metastasis. The 
treatment of metastatic NSCLC only relied on palliative chemoradiotherapy (7) in the past. Surgical resection remains the most effective treatment for patients with early NSCLC. However, approximately $40 \%$ of patients have metastasis at diagnosis, with poor prognosis, and the median survival is only $8-11$ months (6). The most common metastatic sites are the brain, bone, liver and adrenal gland (8). Distant metastasis is often associated with a poor prognosis, but only approximately $7 \%$ of advanced NSCLC have a single metastasis (9). However, for partial metastatic stage IV NSCLC (oligometastatic lung cancer), a number of studies have shown that long-term survival can be achieved with active treatments including surgery or stereotactic radiotherapy. Therefore, in the middle of the 1990s, Hellman and Weichselbaum (10) proposed the concept of "oligometastasis". Oligometastasis is an intermediate state in the process of tumor metastasis, existing in the transition period between localized primary and extensive metastasis (11); the number of metastatic tumors is less than 5 , and the metastatic organ is specific. Hellman proposed the spectral theory and suggested that the state of oligometastasis reflects the inactive biological behavior of tumor cells in vivo; furthermore, ablation of all known metastases can prolong disease-free survival and even cure patients. This theory has been supported by many clinical experiences. For example, liver resection for patients with colorectal liver metastases and pneumonectomy for lung metastasis of sarcomas improved long-term survival (12). "Oligometastasis" emphasizes the localized tumor burden, which is similar to that of isolated distant metastasis, yet different. The number of metastases or distant metastasis organs can be several, not limited to isolated distant metastasis (13). The published studies are based on the number of metastatic foci in clinical data. The number of metastases is less than 5 , defined as oligometastasis. NSCLC patients require metastatic foci of $1-5$ (with oligometastases). For NSCLC with distant metastasis, surgery, systemic chemotherapy, local radiotherapy, targeted therapy and immunotherapy are usually required. The purpose of this study was to compare the efficacy of local surgical treatment with systematic chemoradiotherapy in treatment of NSCLC with oligometastasis.

In this study, a total of 172 patients with NSCLC with oligometastasis in our hospital from January 2006 to December 2016 were analyzed retrospectively. We compared the effects of local surgical treatment and systematic chemoradiotherapy on survival.

\section{Methods}

\section{Inclusion criteria}

In our Hospital Information System (HIS) system, we searched for cases of primary NSCLC with oligometastasis from 2006 to 2016. The study was approved by the ethics committee of our hospital. Inclusion criteria included primary NSCLC diagnosed pathologically; radical resection or partial resection of primary lung cancer; $1-3$ metastatic nodules outside the primary focus, and not more than 1 metastatic organ (including simultaneity and heterochrony). Both groups had an Eastern Cooperative Oncology Group performance status (ECOG PS) scores of $\leq 2$. Simultaneous oligometastasis was defined as oligometastasis detected within 6 months of the diagnosis of primary lung cancer. All patients enrolled in this study had primary lung cancer with distant metastasis and $1-3$ nodules.

\section{General information}

The protocol of this study was approved by the Ethics Review Committee of the National Clinical Research Center for Cancer/Cancer Hospital , and the number/ ID of the Ethics Approval was NCC2016 JP-01. From January 2006 to December 2016, 172 patients with primary NSCLC with oligometastasis was diagnosed in our hospital. Among them, 82 locals' procedures were performed in the operation group (group A). There were 42 males and 40 females, with an average age of $56.4 \pm 11.6$ years old. Pathological diagnosis included adenocarcinoma in 29 cases, squamous cell carcinoma in 47 cases and large cell carcinoma in 6 cases. There were 37 cases of head metastasis, 29 cases of bone metastasis, 9 cases of adrenal metastasis, 2 cases of liver metastasis and 5 cases of other metastasis (four of which had skin metastases and one had ovarian metastases; Table 1). The other 90 cases were treated with radiotherapy and chemotherapy (group B). There were 36 males and 54 females, with an average age of $56.2 \pm 10$.6 years old. Pathological diagnosis included adenocarcinoma in 47 cases, squamous cell carcinoma in 39 cases and large cell carcinoma in 4 cases. There were 37 cases of head metastasis, 22 cases of bone metastasis, 7 cases of adrenal metastasis, 18 cases of liver metastasis, and 6 cases of other metastasis (six patients with oligometastasis in other parts of the radiotherapy and chemotherapy group were treated with local excision of oligometastases; Table 2). For the surgical group, radical resection or partial resection of primary 
Table 1 Clinical characteristics for the surgery group

\begin{tabular}{lc}
\hline Variables & No. of patients $(\mathrm{n}=82)$ \\
\hline Gender & 42 \\
Male & 40 \\
Female & $56.4 \pm 11.6$ \\
Average age & \\
Histology & 47 \\
Squamous cell carcinoma & 29 \\
Adenocarcinoma & 6 \\
Large cell carcinoma & \\
Oligometastatic site & 37 \\
Brain & 29 \\
Bone & 9 \\
Adrenal & 28 \\
Liver & 54 \\
Other & 5 \\
Surgical approach & \\
Pulmonary wedge resection & \\
Lobectomy resection & \\
\hline
\end{tabular}

lung cancer was performed. Adjuvant chemotherapy was recommended after operation. Thirty-seven patients had oligometastasis to the head; 24 of these underwent primary resection of the lung after the partial resection of the oligometastasis, and 13 underwent primary lung resection after head radiotherapy. Of 29 cases of oligometastasis of bone, 24 were transferred in 2-3 cases. After the resection of the primary lung, local radiotherapy was given to the oligometastases, of which 5 cases were rib metastases (all single metastasis cases) that were excised with the primary lung and the resection of the ribs. For 9 patients with adrenal metastases, the primary lung lesions were removed after adrenalectomy. For 2 patients with hepatic metastases, local interventional therapy was performed after resection of primary lung tumor. Five patients ( 4 cases of skin metastases and 1 case of ovarian metastasis) were treated with partial resection of the metastatic lesion and primary resection of the lung. There were 6 cases of oligometastases to other parts of the lung and chemotherapy group.

For patients with more than 1 lesion, bone metastasis occurred in multiple sites, and there were fewer than 3 bone
Table 2 Clinical characteristics for the chemotherapy and radiotherapy group

\begin{tabular}{lc}
\hline Variables & No. of patients $(n=90)$ \\
\hline Gender & 36 \\
Male & 54 \\
Female & $56.2 \pm 10.6$ \\
Average age & \\
Histology & 39 \\
Squamous cell carcinoma & 47 \\
Adenocarcinoma & 4 \\
Large cell carcinoma & \\
Oligometastatic site & 37 \\
Brain & 22 \\
Bone & 7 \\
Adrenal & 18 \\
Liver & 6 \\
Other & \\
\hline
\end{tabular}

metastases . Metastasis removal was performed for a single resectable bone metastasis, and the rest were given local radiotherapy.

\section{Research methods and follow-up}

The enrolled cases were divided into groups A and B. In group A, depending on the patient's lung function and body condition, the surgical method was wedge resection or lobectomy, and adjuvant chemotherapy was recommended after surgery. The patients in group B met the inclusion criteria, but they had poor physical performance and were unable to tolerate surgery, or the patients themselves and/ or their family members refused the surgery. Therefore, they were treated with systemic chemotherapy combined with local radiotherapy. The patients were followed up for 12 months, including by telephone follow-up, medical records and outpatient imaging data.

For patients with peripheral tumors less than $2 \mathrm{~cm}$ in diameter, or with poor or poor lung function or good cancer differentiation, patients with lower malignancy were treated with a wedge resection of the primary lung. Pulmonary resection was reserved for primary tumors, and partial excision was performed at the oligometastatic site. 


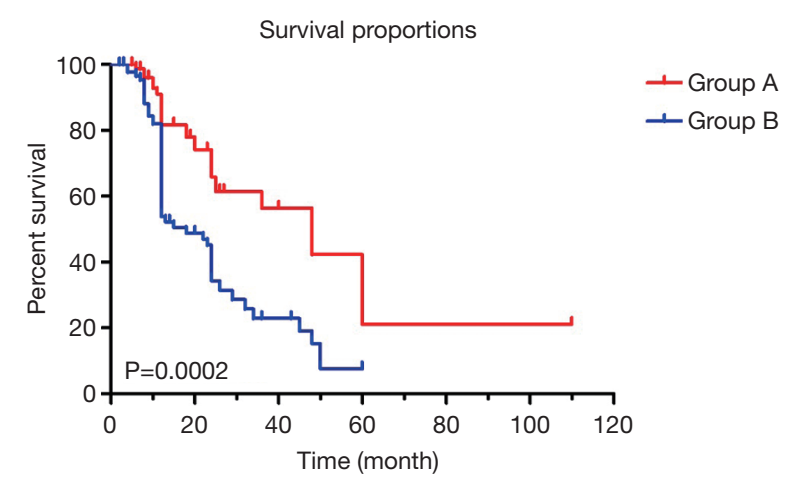

Figure 1 The Kaplan-Meier curve of overall survival time for groups $\mathrm{A}$ and $\mathrm{B}$.

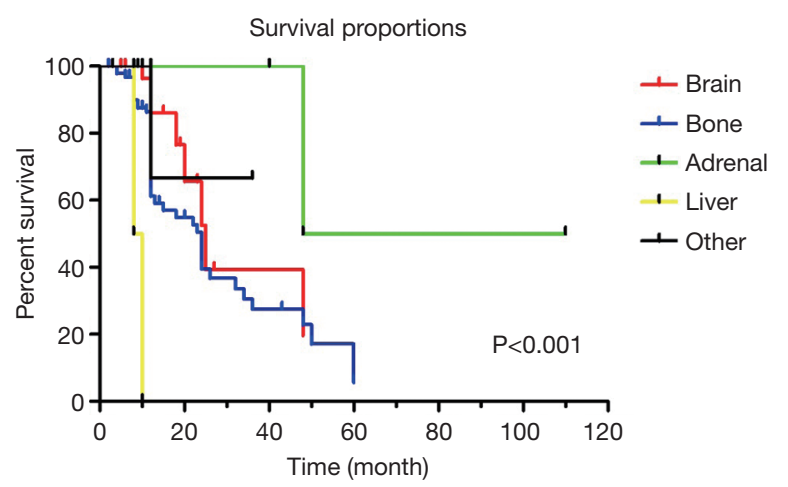

Figure 2 The Kaplan-Meier curve of overall survival time for oligometastatic sites.

\section{Statistical analysis}

SPSS 18 (SPSS, Inc., Chicago, IL, USA) statistical software was used for data processing. Paired $t$-tests were used for the comparison of measurement data. Rank data were compared by rank sum test. The Kaplan-Meier method was used to calculate the survival rate. Differences were statistically significant at $\mathrm{P}<0.05$.

\section{Results}

\section{Surgery methods}

In group A of 82 patients, 54 underwent wedge resection of pulmonary primary tumor, and no severe complications occurred in any patient. Adjuvant chemotherapy was given after the operation. Pulmonary lobectomy and mediastinal lymph node dissection were performed in 28 patients, and no severe complications occurred in any patient. Adjuvant

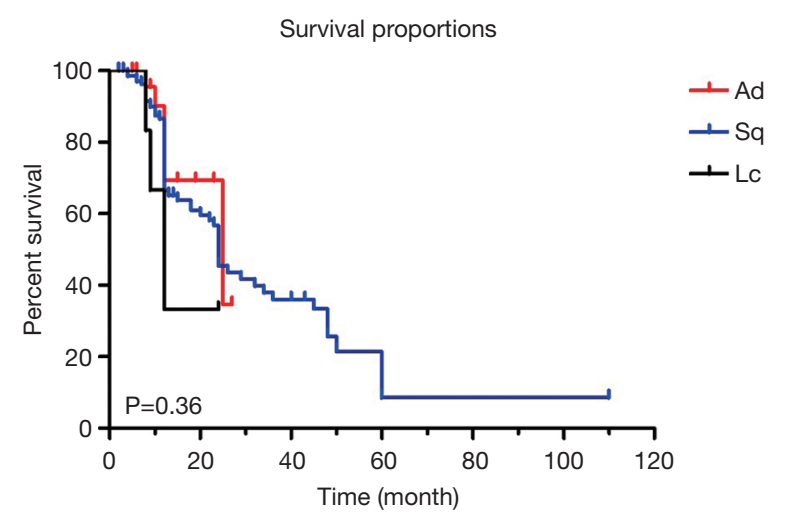

Figure 3 The Kaplan-Meier curve of overall survival time for pathological types. Ad, adenocarcinoma; Sq, squamous cell carcinoma; Lc, large cell carcinoma.

chemotherapy was given after the operation. In group B, 90 patients were treated with systematic radiotherapy and chemotherapy.

\section{Survival status and survival rate}

The two groups were followed up for 12-72 months. There were no statistically significant differences in terms of gender, age, type of pathology or surgery methods of primary tumors $(\mathrm{P}>0.05)$. The median survival time (MST) was 48 months and the 5 -year survival rate were $21.1 \%$ in group A of 82 patients; in group B, the MST was 18 months, and the 5 -year survival rate was $7.6 \%$. The MST and 5 -year survival rates in group A were significantly higher than those of group B $(\mathrm{P}=0.0002)$ (Figure 1). Survival rates were higher in patients with adrenal metastasis than in those with brain, bone, liver, or other metastases $(\mathrm{P}<0.001)$ (Figure 2). There was no significant difference in the survival rate between the pathological type of primary tumor and the surgical methods $(\mathrm{P}=0.36, \mathrm{P}=0.77)$ (Figures 3,4). Our study found that after follow-up of the patient's metastases, approximately $20 \%$ were slightly advanced, and approximately $80 \%$ were controlled. In this study, there were 12 cases with tumor size $\leq 2 \mathrm{~cm}, 42$ cases with tumor size between 2 and $4 \mathrm{~cm}$ and 28 cases with tumor size above $4 \mathrm{~cm}$. We further developed a Cox survival curve for tumor size. The results were not statistically significant $(\mathrm{P}>0.05)$.

\section{Discussion}

There remains controversy as to whether local treatment 


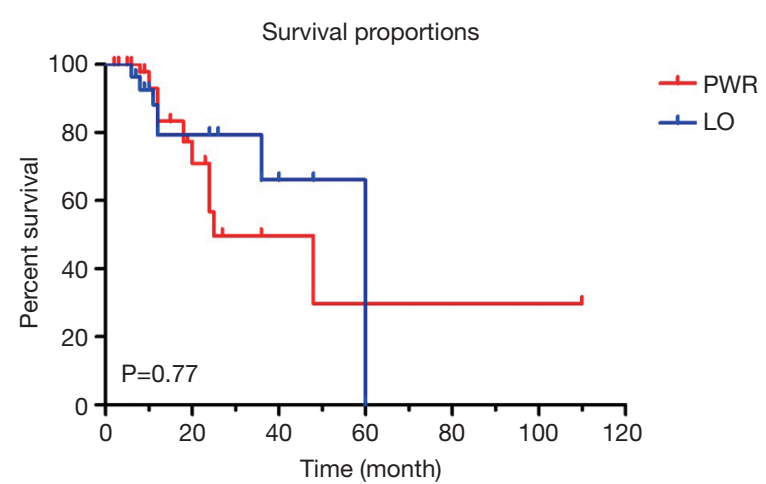

Figure 4 The Kaplan-Meier curve of overall survival time for surgical approach. PWR, pulmonary wedge resection; LO, lobectomy.

of NSCLC with oligometastasis is appropriate. However, most studies reported that treatment of oligometastasis was different from traditional palliative treatment for advanced lung cancer; the relatively limited metastasis therapy highlighted the clinical value of local therapy. In 2015, in order to better guide management, the International Association for the study of lung cancer (IASLC) proposed the eighth edition of NSCLC staging system. The staging guidelines pointed out that oligometastasis was classified as M1b, with prognosis similar to that of M1a, is clearly superior to that of patients with extensive metastatic M1c. Moreover, the new staging guidelines emphasized the advantages of local therapies (such as surgical resection and stereotactic radiotherapy) in the treatment of metastatic lung cancer (14).

NSCLC patients with localized lymph node metastasis were also recommended for different treatment by the eighth edition of the TNM (tumor-node-metastasis) staging system. N1 was subdivided into N1a (single station metastasis) and N1b (multistation metastasis); N2 was subdivided into N2a1 (no N1 metastasis, direct skip to N2 lymph node), N2a2 (N1 lymph node metastasis, simultaneous single station $\mathrm{N} 2$ lymph node metastasis) and N2b (multistation N2 lymph node metastasis) (15). Lymph node metastasis was the most important prognostic risk factor. De Leyn et al. (16) studied 66 patients with bilateral synchronous lung cancer who underwent radical resection. The overall MST was 25.4 months, and the 5-year survival rate was $38 \%$. Although the histopathological types of the subjects were different, there was no significant difference in survival rates among the various tissue types or the same type of multiple primary cancers. Therefore, if there is no evidence of lymph node involvement or distant metastasis, surgical resection is recommended, even if the lung is oligometastatic to the side of the lung. The effect was better than that of stage IV systemic palliative chemotherapy. Endo et al. (17) reported a NSCLC patient with stage I or stage II of thoracic clinical staging who had an oligometastasis. The overall 5 -year survival rate was $40 \%$ after resection. Luketich and Burt (18) compared chemotherapy alone with surgical resection after chemotherapy in NSCLC patients with isolated adrenal metastasis. The MST was 8.5 months in the 6 patients treated with chemotherapy alone, and the MST was 31 months in 8 patients treated with surgical resection after chemotherapy. There was a significant difference, and all patients treated with chemotherapy alone died within 21 months.

NSCLC with oligometastasis is clinically evaluated as the advanced stage of lung cancer, but it is different from advanced lung cancer in general. Whether in terms of biological behavior or treatment strategy, it is very different from traditional advanced lung cancer. Because the number of NSCLC patients with oligometastasis is limited, there remain many controversies regarding the treatment mode of NSCLC oligometastasis. Most of the reports have been retrospective studies, and the results should be validated with prospective studies. For patients with oligometastasis, more active treatment may lead to longer survival in this group of patients.

In this study, the choice of patients was considered in the selection of cases. The choice of bias was mainly gender, age, pathological stage and loss to follow-up rate. There was no significant difference in terms of gender or age between the groups. The loss to follow-up rate was controlled within $5 \%$. For the operation group, primary lung cancer radical resection or partial resection was performed, and adjuvant chemotherapy was recommended after surgery. For the 37 patients with head oligometastasis, 24 underwent partial resection of oligometastasis and underwent surgical resection of the primary lung. Thirteen patients underwent head radiotherapy and underwent surgical resection of the primary lung. Twenty-nine patients with bone oligometastasis, including 24 patients with 2-3 metastases, underwent surgical resection of the primary lung, local radiotherapy, and 5 cases of rib metastasis (all single metastasis cases) were given to the lungs. Surgical resection included primary tumor and resection of rib oligometastases. In 9 patients with adrenal 
oligometastasis, the primary lung was surgically removed in the adrenalectomy. For the 2 patients with hepatic oligometastasis, partial interventional treatment of the liver after removal of the primary lung was performed. For 5 patients with other sites of oligometastasis (4 skin metastases, 1 ovarian metastasis), all patients underwent local excision of the metastatic lesions and then underwent surgical resection of the primary lung. In the radiotherapy and chemotherapy group, intrapulmonary lesions and oligometastases were treated with local radiotherapy plus systemic chemotherapy, and 6 oligometastasis were in other sites (both skin metastasis and partial excision of oligometastases).

There remain many problems to be solved. This study found that local surgery could benefit patients with NSCLC oligometastasis and determined prognostic factors, with certain directive significance for the choice of operative indications. In 82 cases, after the local primary resection was performed, the MST was 48 months, and the survival rate at 5 years was $21.1 \%$. The prognosis was substantially better than that of chemoradiotherapy group, and it was related to the sites of oligometastasis. Patients with adrenal metastasis had better prognoses. However, there were few cases in this study. Metastatic sites did affect overall survival, and patients with adrenal oligometastases had higher survival rates than did patients with brain, bone, liver or other oligometastases. In addition, patients had a recurrence of the lung cancer as well. Because the collected cases are medical records from over nearly 10 years, multimodal treatment, such as targeted therapy and immunotherapy, was not popular enough in the early years, therefore we did not decide on multimodal therapy for these patients. In this study, the choice of patients was considered in the selection of cases. The choice of bias was primarily gender, age, pathological stage and loss to follow-up rate. There was no significant difference in terms of gender and age between the two groups. The loss of follow-up rate was controlled within $5 \%$. Metastatic sites id affects overall survival, and patients with adrenal oligometastases had a higher survival rate than did those with brain, bone, liver or other oligometastases. Due to the small sample size in this study, no further stratified analysis of the metastatic site was performed. In future studies, it is expected that the sample size can be further expanded, and the analysis of the metastatic sites may be done to investigate the long-term outcomes of lung cancer surgery associated with oligometastasis through comparison of metastatic sites.

\section{Acknowledgements}

None.

\section{Footnote}

Conflicts of Interest: The authors have no conflicts of interest to declare.

Ethical Statement: The protocol of this study was approved by the Ethics Review Committee of the National Clinical Research Center for Cancer/Cancer Hospital, and the number/ID of the Ethics Approval was NCC2016 JP-01.

\section{References}

1. Sato J, Horinouchi H, Goto Y, et al. Long-term survival without surgery in NSCLC patients with synchronous brain oligometastasis: systemic chemotherapy revisited. J Thorac Dis 2018;10:1696-702.

2. Noh JM, Ahn YC. How aggressive one need to be in treatment of patients with oligometastasis from non-small cell lung cancer? J Thorac Dis 2017;9:E378-80.

3. He J, Li Y, An J, et al. Surgical treatment in non-small cell lung cancer with pulmonary oligometastasis. World J Surg Oncol 2017;15:36.

4. Suzuki H, Yoshino I. Approach for oligometastasis in non-small cell lung cancer. Gen Thorac Cardiovasc Surg 2016;64:192-6.

5. Siegel RL, Miller KD, Jemal A. Cancer Statistics, 2017. CA Cancer J Clin 2017;67:7-30.

6. Grossi F, Kubota K, Cappuzzo F, et al. Future scenarios for the treatment of advanced non-small cell lung cancer: focus on taxane-containing regimens. Oncologist 2010;15:1102-12.

7. Kawano D, Yano T, Shoji F, et al. The influence of intracellular epidermal growth factor receptor (EGFR) signal activation on the outcome of EGFR tyrosine kinase inhibitor treatment for pulmonary adenocarcinoma. Surg Today 2011;41:818-23.

8. Ramalingam S, Belani C. Systemic chemotherapy for advanced non-small cell lung cancer: recent advances and future directions. Oncologist 2008;13 Suppl 1:5-13.

9. Pfannschmidt J, Dienemann H. Surgical treatment of oligometastatic non-small cell lung cancer. Lung Cancer 2010;69:251-8.

10. Hellman S, Weichselbaum RR. Oligometastases. J Clin Oncol 1995;13:8-10. 
11. Shimada Y, Saji H, Kakihana M, et al. Survival outcomes for oligometastasis in resected non-small cell lung cancer. Asian Cardiovasc Thorac Ann 2015;23:937-44.

12. Yano T, Yasumoto K, Nagashima A, et al. Immunohistological characterization of human monoclonal antibody against lung cancer. J Surg Oncol 1988;39:108-13.

13. Weichselbaum RR, Hellman S. Oligometastases revisited. Nat Rev Clin Oncol 2011;8:378-82.

14. Eberhardt WE, Mitchell A, Crowley J, et al. The IASLC Lung Cancer Staging Project: Proposals for the Revision of the M Descriptors in the Forthcoming Eighth Edition of the TNM Classification of Lung Cancer. J Thorac Oncol 2015;10:1515-22.

15. Ye B, Zhao H. Revision of the TNM Stage Grouping

Cite this article as: Wang Z, Gao SG, Xue Q, Guo XT, Wang LX, Yu X, Yang YK, Mu JW. Surgery of primary non-small cell lung cancer with oligometastasis: analysis of 172 cases. J Thorac Dis 2018;10(12):6540-6546. doi: 10.21037/jtd.2018.11.125 in the Forthcoming Eighth Edition of the TNM Classification for Lung Cancer. Zhongguo Fei Ai Za Zhi 2016;19:337-42.

16. De Leyn P, Moons J, Vansteenkiste J, et al. Survival after resection of synchronous bilateral lung cancer. Eur J Cardiothorac Surg 2008;34:1215-22.

17. Endo C, Hasumi T, Matsumura Y, et al. A prospective study of surgical procedures for patients with oligometastatic non-small cell lung cancer. Ann Thorac Surg 2014;98:258-64.

18. Luketich JD, Burt ME. Does resection of adrenal metastases from non-small cell lung cancer improve survival? Ann Thorac Surg 1996;62:1614-6. 